$\mathrm{DTP} / 99 / 106$

hep-ph/9912251

\title{
ThE TWO-LOOP SCALAR AND TENSOR PENTABOX GRAPH WITH LIGHT-LIKE LEGS
}

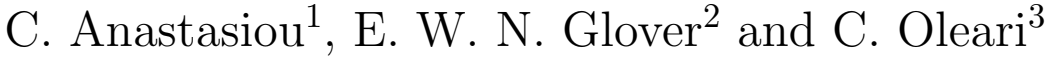 \\ Department of Physics, University of Durham, Durham DH1 3LE, England
}

\begin{abstract}
We study the scalar and tensor integrals associated with the pentabox topology: the class of two-loop box integrals with seven propagators - five in one loop and three in the other. We focus on the case where the external legs are light-like and use integration-by-parts identities to express the scalar integral in terms of two mastertopology integrals and present an explicit analytic expression for the pentabox scalar integral as a series expansion in $\epsilon=(4-D) / 2$. We also give an algorithm based on integration by parts for relating the generic tensor integrals to the same two master integrals and provide general formulae describing the master integrals in arbitrary dimension and with general powers of propagators.
\end{abstract}

PACS: 12.38.Bx, 12.20.Ds, 11.10.Kk

KEYWORDS: scalar integrals, tensor reduction, two-loop integrals, pentabox, dimensional regularisation.

\footnotetext{
${ }^{1}$ e-mail: Ch. Anastasiou@durham.ac.uk

${ }^{2}$ e-mail: E.W.N.Glover@durham.ac.uk

${ }^{3}$ e-mail: Carlo.Oleari@durham.ac.uk
} 


\section{Introduction}

Scattering processes are one of the most important sources of information on short distance physics and have played a vital role in establishing the properties of the fundamental interactions of nature. At the one-loop level, box integrals are a key ingredient in the comparison of perturbative predictions with experimental data, such as wide-angle jet production in hadron collisions or Bhabha scattering in electron-positron annihilation. Recent improvements of experimental measurements demand even more precise theoretical predictions and there is significant interest in determining $2 \rightarrow 2$ scattering rates at the two-loop order. Achieving this goal requires the evaluation of two-loop graphs such as the planar double-box graph [1, 2], the non-planar double-box graph [3] or some one-loop box integrals with bubble insertions on one of the propagators $[4$.

In addition, we also need to study the particular type of planar box graph shown in Fig. 1. Owing to the particular shape of this diagram, we name this topology pentabox. It is the purpose of this paper to provide analytic expressions for the scalar pentabox with unit powers of the propagators using dimensional regularisation for the light-like (or onshell) case, $p_{1}^{2}=p_{2}^{2}=p_{3}^{2}=p_{4}^{2}=0$, in terms of generalised polylogarithms. This type of integral is much simpler than the planar double-box [1] and the non-planar double-box [3] because of the presence of the triangle sub-graph which allows the use of integration-byparts identities [5] to relate the most general scalar integral to two types of simpler integrals with fewer propagators, which we denote master topologies. We give expressions for these simpler integrals and use them to derive an analytic expression for the pentabox graph with unit propagators as a series expansion in $\epsilon=(4-D) / 2$. We also give algorithms for evaluating all the associated tensor integrals. These are directly related to scalar integrals with higher powers of the propagators in higher dimension. As with the scalar integral, the integration-by-parts identities allow the tensor integrals to be obtained in terms of the two master-topology integrals.

Our paper is organised as follows. In Section 2 we establish the basic notation we will use for two-loop integrals. Analytic expressions for the scalar pentabox integral, together with algorithms for reducing the integral to the simpler master topologies, are given in Section 3, while the tensor integrals are described in Section 4 . Details of the master topologies and algorithms relating them are given in Section 5. Explicit expansions in $\epsilon=(4-D) / 2$ are computed in terms of generalised polylogarithm functions. Useful relations amongst the polylogarithms are collected in Appendix A. Finally, our findings are summarized in Section 6 . 


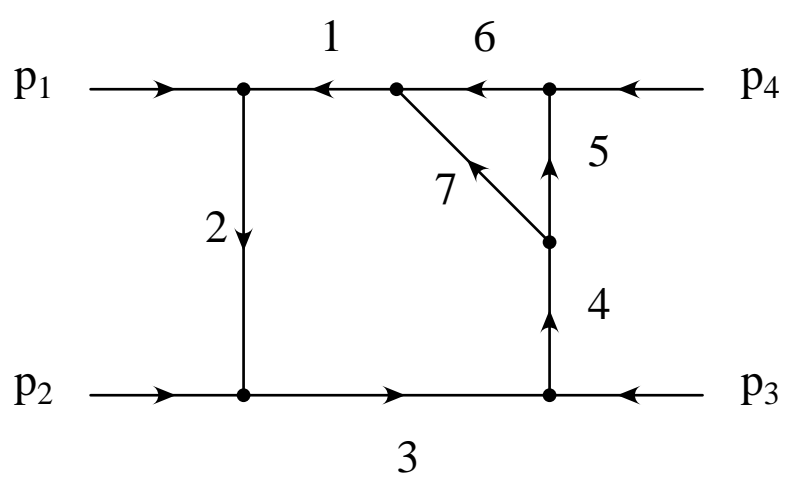

Figure 1: The pentabox topology. The propagators are labelled according to Eq. 2.3) and are each raised to the $\nu_{i}$ power.

\section{Notation}

We denote the generic two-loop integral in $D$ dimensions with $n$ propagators $A_{i}$ raised to arbitrary powers as

$$
J^{D}\left(\left\{\nu_{i}\right\} ;\left\{Q_{i}^{2}\right\}\right)\left[1 ; k_{1}^{\mu} ; k_{2}^{\mu} ; k_{1}^{\mu} k_{1}^{\nu} ; k_{1}^{\mu} k_{2}^{\nu} ; \ldots\right]=\int \frac{d^{D} k_{1}}{i \pi^{D / 2}} \int \frac{d^{D} k_{2}}{i \pi^{D / 2}} \frac{\left[1 ; k_{1}^{\mu} ; k_{2}^{\mu} ; k_{1}^{\mu} k_{1}^{\nu} ; k_{1}^{\mu} k_{2}^{\nu} ; \ldots\right]}{A_{1}^{\nu_{1}} \cdots A_{n}^{\nu_{n}}},
$$

where the external momentum scales are indicated by $\left\{Q_{i}^{2}\right\}$. When the numerator in Eq. (2.1) is unity, $J^{D}\left(\left\{\nu_{i}\right\} ;\left\{Q_{i}^{2}\right\}\right)[1] \equiv J^{D}\left(\left\{\nu_{i}\right\} ;\left\{Q_{i}^{2}\right\}\right)$, we have the scalar integral that forms the basic underlying skeleton of the graph. The tensor integrals $J^{D}\left(\left\{\nu_{i}\right\} ;\left\{Q_{i}^{2}\right\}\right)\left[k_{1}^{\mu} ; \ldots\right]$, which naturally arise in Feynman diagrams, are usually related to combinations of the scalar integral with different powers of propagators and/or different values of $D$ [6]. Evaluation of two-loop matrix elements for physical processes requires a knowledge of both tensor and scalar integrals.

In this paper we focus on the specific two-loop box graph shown in Fig. 1, whose analytic expression is

$$
J^{D}\left(\nu_{1}, \nu_{2}, \nu_{3}, \nu_{4}, \nu_{5}, \nu_{6}, \nu_{7} ; s, t\right)=\int \frac{d^{D} k_{1}}{i \pi^{D / 2}} \int \frac{d^{D} k_{2}}{i \pi^{D / 2}} \frac{1}{A_{1}^{\nu_{1}} A_{2}^{\nu_{2}} A_{3}^{\nu_{3}} A_{4}^{\nu_{4}} A_{5}^{\nu_{5}} A_{6}^{\nu_{6}} A_{7}^{\nu_{7}}},
$$

together with the associated tensor integrals, where the propagators are given by

$$
\begin{aligned}
& A_{1}=k_{1}^{2}+i 0, \\
& A_{2}=\left(k_{1}+p_{1}\right)^{2}+i 0, \\
& A_{3}=\left(k_{1}+p_{1}+p_{2}\right)^{2}+i 0,
\end{aligned}
$$




$$
\begin{aligned}
& A_{4}=\left(k_{1}+p_{1}+p_{2}+p_{3}\right)^{2}+i 0, \\
& A_{5}=\left(k_{2}+p_{1}+p_{2}+p_{3}\right)^{2}+i 0, \\
& A_{6}=k_{2}^{2}+i 0 \\
& A_{7}=\left(k_{1}-k_{2}\right)^{2}+i 0 .
\end{aligned}
$$

With this momentum assignment we see that the loop momentum $k_{1}$ circulates in five of the propagators while $k_{2}$ circulates in only three. All of the external momenta are in-going and are taken to be light-like: $p_{1}^{2}=p_{2}^{2}=p_{3}^{2}=p_{4}^{2}=0$. The only momentum scales present in the problem are therefore the usual Mandelstam variables $s=\left(p_{1}+p_{2}\right)^{2}$ and $t=\left(p_{2}+p_{3}\right)^{2}$, together with $u=-s-t$.

\section{The scalar pentabox integral}

The generic scalar pentabox integral, $J^{D}\left(\nu_{1}, \nu_{2}, \nu_{3}, \nu_{4}, \nu_{5}, \nu_{6}, \nu_{7} ; s, t\right)$, with arbitrary powers of propagators in $D$ dimension is always reducible to a finite set of simpler mastertopology integrals. In fact, applying the integration-by-parts method [5] to the following two integrals

$$
\int \frac{d^{D} k_{1}}{i \pi^{D / 2}} \int \frac{d^{D} k_{2}}{i \pi^{D / 2}} \frac{\partial}{\partial k_{2}^{\mu}} \frac{\left[\left(k_{2}-p_{4}\right)^{\mu} ; k_{2}^{\mu}\right]}{A_{1}^{\nu_{1}} A_{2}^{\nu_{2}} A_{3}^{\nu_{3}} A_{4}^{\nu_{4}} A_{5}^{\nu_{5}} A_{6}^{\nu_{6}} A_{7}^{\nu_{7}}}=0
$$

we find that

$$
\begin{aligned}
& \left(D-2 \nu_{5}-\nu_{6}-\nu_{7}\right) J^{D}=\left(\nu_{6} \mathbf{6}^{+} \mathbf{5}^{-}+\nu_{7} \mathbf{7}^{+} \mathbf{5}^{-}-\nu_{7} \mathbf{7}^{+} \mathbf{4}^{-}\right) J^{D}, \\
& \left(D-\nu_{5}-2 \nu_{6}-\nu_{7}\right) J^{D}=\left(\nu_{5} \mathbf{5}^{+} \boldsymbol{6}^{-}+\nu_{7} \boldsymbol{7}^{+} \boldsymbol{6}^{-}-\nu_{7} \boldsymbol{7}^{+} \mathbf{1}^{-}\right) J^{D},
\end{aligned}
$$

where $J^{D}=J^{D}\left(\nu_{1}, \nu_{2}, \nu_{3}, \nu_{4}, \nu_{5}, \nu_{6}, \nu_{7} ; s, t\right)$ and the symbols $\mathbf{i}^{+}$and $\mathbf{i}^{-}$are shorthand notation for raising and lowering of powers of propagator $i$

$$
\mathbf{i}^{ \pm} J^{D}\left(\ldots, \nu_{i}, \ldots\right)=J^{D}\left(\ldots, \nu_{i} \pm 1, \ldots\right) .
$$

As usual, each raising operator is always accompanied by a factor of $\nu_{i}$ so that it is impossible to raise the power of the propagator if it is not already present, i.e. $\nu_{i} \neq 0$.

By repeated application of Eq. (3.2), we can reduce either of $\nu_{4}$ or $\nu_{5}$ to zero. Similarly, by applying Eq. (3.3) we can lower (and eventually eliminate) the power of either $\nu_{1}$ or $\nu_{6}$. This produces a pinched graph of the form

$$
\begin{aligned}
& J^{D}\left(\nu_{1}, \nu_{2}, \nu_{3}, \nu_{4}, 0,0, \nu_{7} ; s, t\right)=0 \\
& J^{D}\left(\nu_{1}, \nu_{2}, \nu_{3}, 0, \nu_{5}, 0, \nu_{7} ; s, t\right)=A^{D}\left(\nu_{1}, \nu_{2}, \nu_{3}, \nu_{5}, \nu_{7} ; s, t\right), \\
& J^{D}\left(0, \nu_{2}, \nu_{3}, \nu_{4}, 0, \nu_{6}, \nu_{7} ; s, t\right)=B^{D}\left(\nu_{2}, \nu_{3}, \nu_{4}, \nu_{6}, \nu_{7} ; s, t\right), \\
& J^{D}\left(0, \nu_{2}, \nu_{3}, 0, \nu_{5}, \nu_{6}, \nu_{7} ; s, t\right)=C^{D}\left(\nu_{2}, \nu_{3}, \nu_{5}, \nu_{6}, \nu_{7} ; s, t\right),
\end{aligned}
$$


where, diagrammatically,
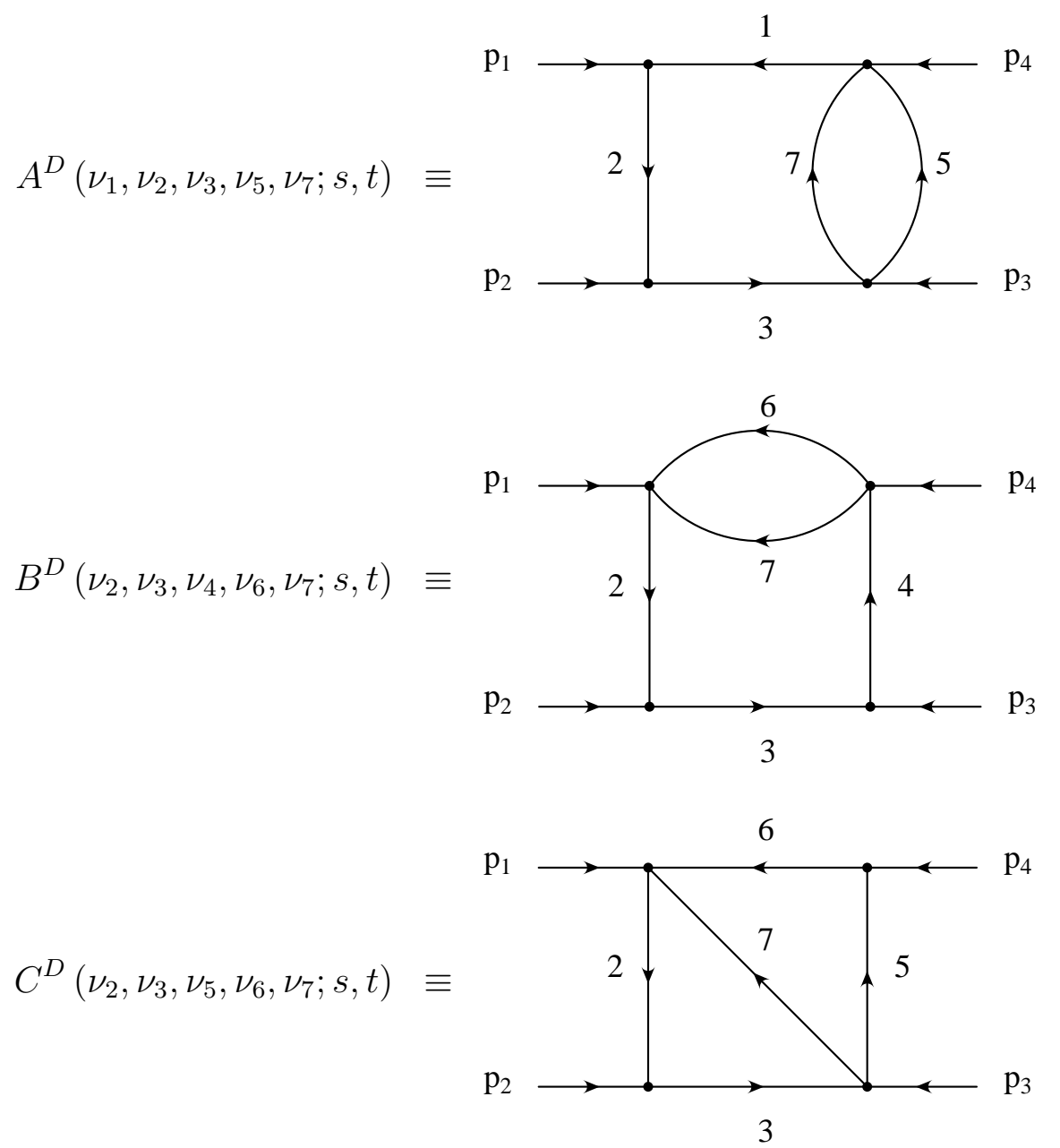

$A^{D}, B^{D}$ and $C^{D}$ constitute our set of master-topology integrals. Due to the particular symmetry of these diagrams, they satisfy some additional identities:

- The basic $A^{D}$ and $B^{D}$ integrals are related by exchanging $s$ and $t$ according to

$$
B^{D}\left(\nu_{2}, \nu_{3}, \nu_{4}, \nu_{6}, \nu_{7} ; s, t\right)=A^{D}\left(\nu_{2}, \nu_{3}, \nu_{4}, \nu_{6}, \nu_{7} ; t, s\right)
$$

and $A^{D}$ has the additional symmetries

$$
\begin{aligned}
& A^{D}\left(\nu_{3}, \nu_{2}, \nu_{1}, \nu_{5}, \nu_{7} ; s, t\right)=A^{D}\left(\nu_{1}, \nu_{2}, \nu_{3}, \nu_{5}, \nu_{7} ; s, t\right), \\
& A^{D}\left(\nu_{1}, \nu_{2}, \nu_{3}, \nu_{7}, \nu_{5} ; s, t\right)=A^{D}\left(\nu_{1}, \nu_{2}, \nu_{3}, \nu_{5}, \nu_{7} ; s, t\right) .
\end{aligned}
$$

- Similarly, the basic $C^{D}$ integral has the properties

$$
\begin{aligned}
& C^{D}\left(\nu_{6}, \nu_{5}, \nu_{3}, \nu_{2}, \nu_{7} ; t, s\right)=C^{D}\left(\nu_{2}, \nu_{3}, \nu_{5}, \nu_{6}, \nu_{7} ; s, t\right), \\
& C^{D}\left(\nu_{3}, \nu_{2}, \nu_{6}, \nu_{5}, \nu_{7} ; t, s\right)=C^{D}\left(\nu_{2}, \nu_{3}, \nu_{5}, \nu_{6}, \nu_{7} ; s, t\right) .
\end{aligned}
$$


Application: We consider here the specific case of the scalar integral with all the propagators equal to one. Applying Eqs. (3.2) and (3.3) we obtain

$$
\begin{aligned}
J^{D}(1,1,1,1,1,1,1 ; s, t)=\frac{1}{(D-5)(D-4)} \\
\times \quad\left[2 C^{D}(1,1,1,1,3 ; s, t)-2 A^{D}(1,1,1,1,3 ; s, t)-A^{D}(1,1,1,2,2 ; s, t)\right. \\
\left.\quad-2 B^{D}(1,1,1,1,3 ; s, t)-B^{D}(1,1,1,2,2 ; s, t)\right]
\end{aligned}
$$

The explicit form for the master-topology integrals with arbitrary powers of propagators and arbitrary dimension are collected in Section 5. Making a series expansion in $\epsilon=(4-D) / 2$, we find that the general structure of the scalar integral can be written

$$
\begin{aligned}
J^{D}(1,1,1,1,1,1,1 ; s, t)= & \frac{3}{4 \epsilon^{4}} \frac{(1+3 \epsilon)}{(1+\epsilon)(1+2 \epsilon)} \frac{\Gamma(1-\epsilon)^{3} \Gamma(1+2 \epsilon)}{\Gamma(1-3 \epsilon)} \\
& \times\left[\frac{(-t)^{-2 \epsilon}}{s t^{2}} R(s, t)+\frac{(-s)^{-2 \epsilon}}{t s^{2}} R(t, s)\right] .
\end{aligned}
$$

1) In the physical region $\boldsymbol{s}>\mathbf{0}, \boldsymbol{t}<\mathbf{0}$ we have

$$
\begin{aligned}
R(s, t)= & 1+\epsilon+2 \zeta_{2} \epsilon^{2}+\left[4 \mathrm{~S}_{1,2}\left(\frac{s+t}{s}\right)+2 \zeta_{3}+6 \zeta_{2}\right] \epsilon^{3} \\
& +\left\{12 \mathrm{~S}_{2,2}\left(\frac{s+t}{s}\right)-8 \mathrm{~S}_{1,3}\left(\frac{s+t}{s}\right)+8 \mathrm{~S}_{1,2}\left(\frac{s+t}{s}\right)+28 \zeta_{2} \operatorname{Li}_{2}\left(\frac{s+t}{s}\right)\right. \\
& \left.+6 \zeta_{3}-51 \zeta_{4}\right\} \epsilon^{4}+4 i \pi \Theta(s, t)\left\{\left[\operatorname{Li}_{2}\left(\frac{s+t}{s}\right)-\zeta_{2}\right] \epsilon^{3}\right. \\
& \left.+\left[3 \mathrm{Li}_{3}\left(\frac{s+t}{s}\right)+2 \mathrm{Li}_{2}\left(\frac{s+t}{s}\right)-2 \mathrm{~S}_{1,2}\left(\frac{s+t}{s}\right)-\zeta_{3}-2 \zeta_{2}\right] \epsilon^{4}\right\}+\mathcal{O}\left(\epsilon^{5}\right) .
\end{aligned}
$$

The generalised polylogarithms (defined in Section A.1) that appear in Eq. (3.14), and then in Eq. (3.13), have arguments $(s+t) / t$ and $(s+t) / s$, which are always less than unity in this region. The function $\Theta(t, s)$ assumes values

$$
\Theta(s, t)=-\Theta(t, s)=-1
$$

The imaginary parts associated with the prefactor can be simply obtained by recalling the $+i 0$ prescription associated with the external kinematic scales

$$
\begin{aligned}
& (-s)^{-2 \epsilon} \rightarrow|s|^{-2 \epsilon} \exp (2 \pi i \epsilon), \\
& (-t)^{-2 \epsilon} \rightarrow|t|^{-2 \epsilon} .
\end{aligned}
$$


2) In the other physical region $\boldsymbol{s}<\mathbf{0}, \boldsymbol{t}<\mathbf{0}$, we use the analytic continuation formulae of Section A.2, and we find

$$
\begin{aligned}
R(s, t)= & 1+\epsilon+2 \zeta_{2} \epsilon^{2}+\left[4 \operatorname{Li}_{3}\left(\frac{s}{s+t}\right)+4 \log \left(\frac{s+t}{s}\right) \operatorname{Li}_{2}\left(\frac{s}{s+t}\right)\right. \\
& \left.+\frac{2}{3} \log ^{3}\left(\frac{s+t}{s}\right)+2 \pi^{2} \log \left(\frac{s+t}{s}\right)-4 \mathrm{~S}_{1,2}\left(\frac{s}{s+t}\right)+6 \zeta_{3}+\pi^{2}\right] \epsilon^{3} \\
& +\left\{4 \mathrm{~S}_{2,2}\left(\frac{s}{s+t}\right)+8 \mathrm{~S}_{1,3}\left(\frac{s}{s+t}\right)-8\left[\log \left(\frac{s+t}{s}\right)+1\right] \mathrm{S}_{1,2}\left(\frac{s}{s+t}\right)\right. \\
& -16 \operatorname{Li}_{4}\left(\frac{s}{s+t}\right)+4\left[2-\log \left(\frac{s+t}{s}\right)\right] \operatorname{Li}_{3}\left(\frac{s}{s+t}\right)+\frac{5}{6} \log ^{4}\left(\frac{s+t}{s}\right) \\
& +4\left[\log ^{2}\left(\frac{s+t}{s}\right)+2 \log \left(\frac{s+t}{s}\right)-\zeta_{2}\right] \operatorname{Li}_{2}\left(\frac{s}{s+t}\right)+\frac{4}{3} \log ^{3}\left(\frac{s+t}{s}\right) \\
& \left.+\frac{8}{3} \pi^{2} \log ^{2}\left(\frac{s+t}{s}\right)+12\left[\zeta_{3}+2 \zeta_{2}\right] \log \left(\frac{s+t}{s}\right)+14 \zeta_{3}+31 \zeta_{4}\right\} \epsilon^{4}+\mathcal{O}\left(\epsilon^{5}\right) .
\end{aligned}
$$

Notice that, in this region, the scalar pentabox integral of Eq. (3.13) has no imaginary part, as expected.

\section{The generic tensor integrals}

In this section, we want to deal with the generic tensor integral, $J^{D}\left(\left\{\nu_{i}\right\} ;\left\{Q_{i}^{2}\right\}\right)\left[k_{1}^{\mu} ; \ldots\right]$, using the pentabox just as an example of how to reduce the tensor integral to a set of simpler scalar integrals.

A method to reduce tensor integrals constructing differential operators that change the powers of the propagators as well as the dimension of the integral was presented in Ref. [6]. However, it is in our view simpler to obtain the tensor integrals directly from the Schwinger parameterised form of the integral. In fact, in its most general form, the integrand can be rewritten as

$$
\frac{1}{A_{1}^{\nu_{1}} \ldots A_{n}^{\nu_{n}}}=\int \mathcal{D} x \exp \left(\sum_{i=1}^{n} x_{i} A_{i}\right)
$$

where

$$
\int \mathcal{D} x=\prod_{i=1}^{n} \frac{(-1)^{\nu_{i}}}{\Gamma\left(\nu_{i}\right)} \int_{0}^{\infty} d x_{i} x_{i}^{\nu_{i}-1}
$$

and

$$
\sum_{i=1}^{n} x_{i} A_{i}=a k_{1}^{2}+b k_{2}^{2}+2 c k_{1} \cdot k_{2}+2 d \cdot k_{1}+2 e \cdot k_{2}+f,
$$

where $a, b, c, d^{\mu}, e^{\mu}$ and $f$ are directly readable from the actual graph: $a(b)=\sum x_{i}$, where the sum runs over the legs in the $k_{1}\left(k_{2}\right)$ loop, and $c=\sum x_{i}$ with the sum running over the 
legs common to both loops. Specifically, in the pentabox graph we have

$$
\begin{aligned}
a & =x_{1}+x_{2}+x_{3}+x_{4}+x_{7} \\
b & =x_{5}+x_{6}+x_{7} \\
c & =-x_{7} \\
d^{\mu} & =x_{2} p_{1}^{\mu}+x_{3} p_{12}^{\mu}+x_{4} p_{123}^{\mu} \\
e^{\mu} & =x_{5} p_{123}^{\mu} \\
f & =x_{3} s,
\end{aligned}
$$

where $p_{12}=p_{1}+p_{2}$ and $p_{123}=p_{1}+p_{2}+p_{3}$. In all cases, the $a, b, c, d^{\mu}, e^{\mu}$ and $f$ are linear in the $x_{i}$.

With the change of variables

$$
\begin{aligned}
k_{1}^{\mu} & \rightarrow K_{1}^{\mu}-\frac{c K_{2}^{\mu}}{a}+\mathcal{X}^{\mu}, \\
k_{2}^{\mu} & \rightarrow K_{2}^{\mu}+\mathcal{Y}^{\mu}
\end{aligned}
$$

where

$$
\mathcal{X}^{\mu}=\frac{c e^{\mu}-b d^{\mu}}{\mathcal{P}}, \quad \mathcal{Y}^{\mu}=\frac{c d^{\mu}-a e^{\mu}}{\mathcal{P}}
$$

and

$$
\mathcal{P}=a b-c^{2}
$$

we can diagonalise Eq. (4.3), so that

$$
\sum_{i=1}^{n} x_{i} A_{i}=a K_{1}^{2}+\frac{\mathcal{P}}{a} K_{2}^{2}+\frac{\mathcal{Q}}{\mathcal{P}}
$$

with

$$
\mathcal{Q}=-a e^{2}-b d^{2}+2 c e \cdot d+f \mathcal{P} .
$$

The scalar loop integral can be cast in the form

$$
J^{D}\left(\left\{\nu_{i}\right\} ;\left\{Q_{i}^{2}\right\}\right)[1]=\int \mathcal{D} x \int \frac{d^{D} K_{1}}{i \pi^{D / 2}} \int \frac{d^{D} K_{2}}{i \pi^{D / 2}} \exp \left[a K_{1}^{2}+\frac{\mathcal{P}}{a} K_{2}^{2}+\frac{\mathcal{Q}}{\mathcal{P}}\right],
$$

and the Gaussian integrals over the shifted loop momenta are evaluated to

$$
J^{D}\left(\left\{\nu_{i}\right\} ;\left\{Q_{i}^{2}\right\}\right)[1]=\int \mathcal{D} x \mathcal{I}
$$

the integrand $\mathcal{I}$ being given by

$$
\mathcal{I}=\frac{1}{\mathcal{P}^{D / 2}} \exp \left(\frac{\mathcal{Q}}{\mathcal{P}}\right)
$$


Similarly, the tensor integrals can be easily obtained by using identities such as

$$
\begin{aligned}
\int \frac{d^{D} K_{1}}{i \pi^{D / 2}} K_{1}^{\mu} \exp \left(a K_{1}^{2}\right) & =0 \\
\int \frac{d^{D} K_{1}}{i \pi^{D / 2}} K_{1}^{\mu} K_{1}^{\nu} \exp \left(a K_{1}^{2}\right) & =-\frac{1}{2 a} g^{\mu \nu} \frac{1}{a^{D / 2}} \\
\int \frac{d^{D} K_{1}}{i \pi^{D / 2}} K_{1}^{\mu} K_{1}^{\nu} K_{1}^{\rho} K_{1}^{\sigma} \exp \left(a K_{1}^{2}\right) & =\frac{1}{4 a^{2}}\left\{g^{\mu \nu} g^{\rho \sigma}+g^{\mu \rho} g^{\nu \sigma}+g^{\mu \sigma} g^{\nu \rho}\right\} \frac{1}{a^{D / 2}} .
\end{aligned}
$$

To give a concrete example, we consider the tensor integral associated with $k_{1}^{\mu}$

$$
\begin{aligned}
J^{D}\left(\left\{\nu_{i}\right\} ;\left\{Q_{i}^{2}\right\}\right)\left[k_{1}^{\mu}\right] & =\int \mathcal{D} x \\
& \times \int \frac{d^{D} K_{1}}{i \pi^{D / 2}} \int \frac{d^{D} K_{2}}{i \pi^{D / 2}}\left\{K_{1}^{\mu}-\frac{c K_{2}^{\mu}}{a}+\mathcal{X}^{\mu}\right\} \exp \left(a K_{1}^{2}+\frac{\mathcal{P}}{a} K_{2}^{2}+\frac{\mathcal{Q}}{\mathcal{P}}\right) \\
& =\int \mathcal{D} x \mathcal{X}^{\mu} \mathcal{I} .
\end{aligned}
$$

Recalling the definition (4.7), we see that $\mathcal{X}^{\mu}$ consists of the ratio of a set of bilinears in $x_{i}$ divided by $\mathcal{P}$. We can therefore absorb the factors of $x_{i}$ into $\mathcal{D} x$ (see Eq. (4.2)) by increasing the power to which the $i$-th propagator is raised

$$
\frac{(-1)^{\nu_{i}} x_{i}^{\nu_{i}-1}}{\Gamma\left(\nu_{i}\right)} x_{i} \Longrightarrow-\nu_{i} \frac{(-1)^{\nu_{i}+1} x_{i}^{\nu_{i}}}{\Gamma\left(\nu_{i}+1\right)} \equiv-\nu_{i} \mathbf{i}^{+},
$$

while the factor $\mathcal{P}$ can be absorbed into $\mathcal{I}$ (see Eq. (4.13))

$$
\frac{1}{\mathcal{P}^{D / 2}} \frac{1}{\mathcal{P}} \Longrightarrow \frac{1}{\mathcal{P}^{(D+2) / 2}}
$$

so that $1 / \mathcal{P}$ acts as a dimension increaser

$$
\frac{1}{\mathcal{P}} \Longrightarrow \mathbf{d}^{+}, \quad \mathcal{P} \Longrightarrow \mathbf{d}^{-}
$$

where

$$
\mathbf{d}^{ \pm} J^{D}\left(\left\{\nu_{i}\right\} ;\left\{Q_{i}^{2}\right\}\right)=J^{D \pm 2}\left(\left\{\nu_{i}\right\} ;\left\{Q_{i}^{2}\right\}\right) .
$$

In this way, each $x_{i}$ in the numerator increases by one the power of the associated propagator, and each power of $\mathcal{P}$ in the denominator (numerator) increases (decreases) the space-time dimension $D$ by two. Schematically we have

$$
J^{D}\left(\left\{\nu_{i}\right\} ;\left\{Q_{i}^{2}\right\}\right)\left[k_{1}^{\mu}\right]=\sum \nu_{i} \nu_{j} p_{k}^{\mu} J^{D+2}\left(\left\{\ldots, \nu_{i+1}, \ldots, \nu_{j+1}, \ldots\right\} ;\left\{Q_{i}^{2}\right\}\right)[1],
$$

where the summation runs over the elements of $\left(c e^{\mu}-b d^{\mu}\right)$ which fix the values of $i, j$ and $p_{k}$. 
For the case of the pentabox where $a, \ldots, f$ are given in Eq. (4.4), we have from Eq. (4.7)

$$
\mathcal{X}^{\mu}=\frac{-x_{7} x_{5} p_{123}^{\mu}-\left(x_{5}+x_{6}+x_{7}\right)\left(x_{2} p_{1}^{\mu}+x_{3} p_{12}^{\mu}+x_{4} p_{123}^{\mu}\right)}{\mathcal{P}}
$$

so that Eq. (4.17) becomes

$J^{D}\left[k_{1}^{\mu}\right]=\left[-\nu_{5} 5^{+} \nu_{7} \mathbf{7}^{+} p_{123}^{\mu}-\left(\nu_{5} 5^{+}+\nu_{6} \mathbf{6}^{+}+\nu_{7} \mathbf{7}^{+}\right)\left(\nu_{2} \mathbf{2}^{+} p_{1}^{\mu}+\nu_{3} \mathbf{3}^{+} p_{12}^{\mu}+\nu_{4} \mathbf{4}^{+} p_{123}^{\mu}\right)\right] \mathbf{d}^{+} J^{D}$,

where we have used the shorthand notation $J^{D}=J^{D}\left(\left\{\nu_{i}\right\} ;\left\{Q_{i}^{2}\right\}\right)$.

By applying the integration-by-parts identities (3.2) and (3.3) we can now write this tensor integral directly in terms of the set of simpler integrals.

For generic four-point integrals, we need tensor integrals with up to four free indices, each associated with a Lorentz index of an external leg. Integrals with higher powers of the loop momenta are of course possible, but must yield dot products with other momenta when the available free Lorentz indices are saturated. In many cases, these dot products can be immediately expressed in terms of the propagators and canceled through (see Eq. (4.38)).

Nevertheless, the procedure previously described can be iterated ad libitum and we can express every tensor integrals in terms of scalar ones with increased powers of the propagators and dimension $D$. For example, we have

$$
\begin{aligned}
J^{D}\left[k_{2}^{\mu}\right] & =\int \mathcal{D} x \mathcal{Y}^{\mu} \mathcal{I} \\
J^{D}\left[k_{1}^{\mu} k_{1}^{\nu}\right] & =\int \mathcal{D} x\left(\mathcal{X}^{\mu} \mathcal{X}^{\nu}-\frac{b}{2 P} g^{\mu \nu}\right) \mathcal{I} \\
J^{D}\left[k_{1}^{\mu} k_{2}^{\nu}\right] & =\int \mathcal{D} x\left(\mathcal{X}^{\mu} \mathcal{Y}^{\nu}+\frac{c}{2 P} g^{\mu \nu}\right) \mathcal{I} \\
J^{D}\left[k_{2}^{\mu} k_{2}^{\nu}\right] & =\int \mathcal{D} x\left(\mathcal{Y}^{\mu} \mathcal{Y}^{\nu}-\frac{a}{2 P} g^{\mu \nu}\right) \mathcal{I} \\
J^{D}\left[k_{1}^{\mu} k_{1}^{\nu} k_{1}^{\rho}\right] & =\int \mathcal{D} x\left(\mathcal{X}^{\mu} \mathcal{X}^{\nu} \mathcal{X}^{\rho}-\frac{b}{2 \mathcal{P}}\left\{g^{\mu \nu} \mathcal{X}^{\rho}+g^{\mu \rho} \mathcal{X}^{\nu}+g^{\nu \rho} \mathcal{X}^{\mu}\right\}\right) \mathcal{I}, \\
J^{D}\left[k_{1}^{\mu} k_{1}^{\nu} k_{2}^{\rho}\right] & =\int \mathcal{D} x\left(\mathcal{X}^{\mu} \mathcal{X}^{\nu} \mathcal{Y}^{\rho}-\frac{b}{2 \mathcal{P}} g^{\mu \nu} \mathcal{Y}^{\rho}+\frac{c}{2 \mathcal{P}}\left\{g^{\mu \rho} \mathcal{X}^{\nu}+g^{\nu \rho} \mathcal{X}^{\mu}\right\}\right) \mathcal{I}, \\
J^{D}\left[k_{1}^{\mu} k_{2}^{\nu} k_{2}^{\rho}\right] & =\int \mathcal{D} x\left(\mathcal{X}^{\mu} \mathcal{Y}^{\nu} \mathcal{Y}^{\rho}-\frac{a}{2 \mathcal{P}} g^{\nu \rho} \mathcal{X}^{\mu}+\frac{c}{2 \mathcal{P}}\left\{g^{\mu \nu} \mathcal{Y}^{\rho}+g^{\mu \rho} \mathcal{Y}^{\nu}\right\}\right) \mathcal{I}, \\
J^{D}\left[k_{2}^{\mu} k_{2}^{\nu} k_{2}^{\rho}\right] & =\int \mathcal{D} x\left(\mathcal{Y}^{\mu} \mathcal{Y}^{\nu} \mathcal{Y}^{\rho}-\frac{a}{2 \mathcal{P}}\left\{g^{\mu \nu} \mathcal{Y}^{\rho}+g^{\mu \rho} \mathcal{Y}^{\nu}+g^{\nu \rho} \mathcal{Y}^{\mu}\right\}\right) \mathcal{I}, \\
J^{D}\left[k_{1}^{\mu} k_{1}^{\nu} k_{1}^{\rho} k_{1}^{\sigma}\right]= & \int \mathcal{D} x\left(\mathcal{X}^{\mu} \mathcal{X}^{\nu} \mathcal{X}^{\rho} \mathcal{X}^{\sigma}+\frac{b^{2}}{4 \mathcal{P}^{2}}\left\{g^{\mu \nu} g^{\rho \sigma}+g^{\mu \rho} g^{\nu \sigma}+g^{\mu \sigma} g^{\nu \rho}\right\}\right. \\
& -\frac{b}{2 \mathcal{P}}\left\{g^{\mu \nu} \mathcal{X}^{\rho} \mathcal{X}^{\sigma}+g^{\mu \rho} \mathcal{X}^{\nu} \mathcal{X}^{\sigma}+g^{\mu \sigma} \mathcal{X}^{\nu} \mathcal{X}^{\rho}+g^{\nu \rho} \mathcal{X}^{\mu} \mathcal{X}^{\sigma}\right.
\end{aligned}
$$




$$
\begin{aligned}
& \left.\left.+g^{\nu \sigma} \mathcal{X}^{\mu} \mathcal{X}^{\rho}+g^{\rho \sigma} \mathcal{X}^{\mu} \mathcal{X}^{\nu}\right\}\right) \mathcal{I} \\
& J^{D}\left[k_{1}^{\mu} k_{1}^{\nu} k_{1}^{\rho} k_{2}^{\sigma}\right]=\int \mathcal{D} x\left(\mathcal{X}^{\mu} \mathcal{X}^{\nu} \mathcal{X}^{\rho} \mathcal{Y}^{\sigma}-\frac{b}{2 \mathcal{P}}\left\{g^{\mu \nu} \mathcal{X}^{\rho}+g^{\mu \rho} \mathcal{X}^{\nu}+g^{\nu \rho} \mathcal{X}^{\mu}\right\} \mathcal{Y}^{\sigma}\right. \\
& +\frac{c}{2 \mathcal{P}}\left\{g^{\mu \sigma} \mathcal{X}^{\nu} \mathcal{X}^{\rho}+g^{\nu \sigma} \mathcal{X}^{\mu} \mathcal{X}^{\rho}+g^{\rho \sigma} \mathcal{X}^{\mu} \mathcal{X}^{\nu}\right\} \\
& \left.-\frac{b c}{4 \mathcal{P}^{2}}\left\{g^{\mu \nu} g^{\rho \sigma}+g^{\mu \rho} g^{\nu \sigma}+g^{\mu \sigma} g^{\nu \rho}\right\}\right) \mathcal{I}, \\
& J^{D}\left[k_{1}^{\mu} k_{1}^{\nu} k_{2}^{\rho} k_{2}^{\sigma}\right]=\int \mathcal{D} x\left(\mathcal{X}^{\mu} \mathcal{X}^{\nu} \mathcal{Y}^{\rho} \mathcal{Y}^{\sigma}-\frac{a}{2 \mathcal{P}} g^{\rho \sigma} \mathcal{X}^{\mu} \mathcal{X}^{\nu}-\frac{b}{2 \mathcal{P}} g^{\mu \nu} \mathcal{Y}^{\rho} \mathcal{Y}^{\sigma}\right. \\
& +\frac{c}{2 \mathcal{P}}\left\{g^{\mu \rho} \mathcal{X}^{\nu} \mathcal{Y}^{\sigma}+g^{\nu \rho} \mathcal{X}^{\mu} \mathcal{Y}^{\sigma}+g^{\mu \sigma} \mathcal{X}^{\nu} \mathcal{Y}^{\rho}+g^{\nu \sigma} \mathcal{X}^{\mu} \mathcal{Y}^{\rho}\right\} \\
& \left.+\frac{a b}{4 \mathcal{P}^{2}} g^{\mu \nu} g^{\rho \sigma}+\frac{c^{2}}{4 \mathcal{P}^{2}}\left\{g^{\mu \rho} g^{\nu \sigma}+g^{\mu \sigma} g^{\nu \rho}\right\}\right) \mathcal{I} \\
& J^{D}\left[k_{1}^{\mu} k_{2}^{\nu} k_{2}^{\rho} k_{2}^{\sigma}\right]=\int \mathcal{D} x\left(\mathcal{X}^{\mu} \mathcal{Y}^{\nu} \mathcal{Y}^{\rho} \mathcal{Y}^{\sigma}-\frac{a}{2 \mathcal{P}}\left\{g^{\nu \rho} \mathcal{Y}^{\sigma}+g^{\nu \sigma} \mathcal{Y}^{\rho}+g^{\rho \sigma} \mathcal{Y}^{\nu}\right\} \mathcal{X}^{\mu}\right. \\
& +\frac{c}{2 \mathcal{P}}\left\{g^{\mu \nu} \mathcal{Y}^{\rho} \mathcal{Y}^{\sigma}+g^{\mu \rho} \mathcal{Y}^{\nu} \mathcal{Y}^{\sigma}+g^{\mu \sigma} \mathcal{Y}^{\nu} \mathcal{Y}^{\rho}\right\} \\
& \left.-\frac{a c}{4 \mathcal{P}^{2}}\left\{g^{\mu \nu} g^{\rho \sigma}+g^{\mu \rho} g^{\nu \sigma}+g^{\mu \sigma} g^{\nu \rho}\right\}\right) \mathcal{I}, \\
& J^{D}\left[k_{2}^{\mu} k_{2}^{\nu} k_{2}^{\rho} k_{2}^{\sigma}\right]=\int \mathcal{D} x\left(\mathcal{Y}^{\mu} \mathcal{Y}^{\nu} \mathcal{Y}^{\rho} \mathcal{Y}^{\sigma}+\frac{a^{2}}{4 \mathcal{P}^{2}}\left\{g^{\mu \nu} g^{\rho \sigma}+g^{\mu \rho} g^{\nu \sigma}+g^{\mu \sigma} g^{\nu \rho}\right\}\right. \\
& -\frac{a}{2 \mathcal{P}}\left\{g^{\mu \nu} \mathcal{Y}^{\rho} \mathcal{Y}^{\sigma}+g^{\mu \rho} \mathcal{Y}^{\nu} \mathcal{Y}^{\sigma}+g^{\mu \sigma} \mathcal{Y}^{\nu} \mathcal{Y}^{\rho}+g^{\nu \rho} \mathcal{Y}^{\mu} \mathcal{Y}^{\sigma}\right. \\
& \left.\left.+g^{\nu \sigma} \mathcal{Y}^{\mu} \mathcal{Y}^{\rho}+g^{\rho \sigma} \mathcal{Y}^{\mu} \mathcal{Y}^{\nu}\right\}\right) \mathcal{I}
\end{aligned}
$$

Note that these expressions are valid for arbitrary two-loop integrals and to use them all that needs to be done is to identify $a, b, c, d^{\mu}, e^{\mu}$ and $f$, which can easily be read off from the graph, and to construct $\mathcal{X}^{\mu}$ and $\mathcal{Y}^{\mu}$. The powers of $x_{i}$ and $\mathcal{P}$ can then be exchanged for scalar integrals with higher $\nu_{i}$ and higher $D$. This procedure is straightforward to implement in an algebraic program.

Of course, rewriting the tensor integrals in this way is only useful if the relevant scalar integrals in higher $D$ and with arbitrary powers of the propagators are known. For the pentabox, this is the case since repeated application of the integration-by-parts identities (3.2) and (3.3) can always be used to reduce the integrals to one of the master-topology integrals of Eqs. (3.6)-(3.8), for which general results are given in the next section. 
However, there is one further simplification of note. When in the numerator we have a dot product that can be rewritten in terms of the difference of propagators, it is easiest to directly cancel off one of the propagator powers. For example, if we have to deal with $k_{1} \cdot p_{1}$, it is more efficient to make the replacement

$$
2 k_{1} \cdot p_{1}=\left(k_{1}+p_{1}\right)^{2}-k_{1}^{2}=A_{2}-A_{1} \equiv \mathbf{2}^{-}-\mathbf{1}^{-} \text {. }
$$

The integration-by-parts identities (3.2) and (3.3) can still be used to further reduce the powers of $\nu_{1}, \nu_{4}, \nu_{5}$ and $\nu_{6}$.

Following this procedure, we can pinch completely the second propagator, so that we immediately obtain self-energy insertions to one-loop triangle graphs of the type shown in Fig. 2. These can be written directly in terms of $\Gamma$ functions

$$
\begin{aligned}
& J^{D}\left(\nu_{1}, 0, \nu_{3}, 0, \nu_{5}, 0, \nu_{7} ; s, t\right)=\Pi^{D}\left(\nu_{5}, \nu_{7}\right) I_{3}^{D}\left(\nu_{5}+\nu_{7}-\frac{D}{2}, \nu_{1}, \nu_{3} ; s\right), \\
& J^{D}\left(0,0, \nu_{3}, \nu_{4}, 0, \nu_{6}, \nu_{7} ; s, t\right)=\Pi^{D}\left(\nu_{6}, \nu_{7}\right) I_{3}^{D}\left(\nu_{4}, \nu_{6}+\nu_{7}-\frac{D}{2}, \nu_{3} ; s\right), \\
& J^{D}\left(0,0, \nu_{3}, 0, \nu_{5}, \nu_{6}, \nu_{7} ; s, t\right)=\Pi^{D}\left(\nu_{3}, \nu_{7}\right) I_{3}^{D}\left(\nu_{5}, \nu_{3}+\nu_{7}-\frac{D}{2}, \nu_{6} ; s\right),
\end{aligned}
$$

where the self-energy factor is given by

$$
\Pi^{D}\left(\nu_{1}, \nu_{2}\right)=(-1)^{\frac{D}{2}} \frac{\Gamma\left(\nu_{1}+\nu_{2}-\frac{D}{2}\right) \Gamma\left(\frac{D}{2}-\nu_{1}\right) \Gamma\left(\frac{D}{2}-\nu_{2}\right)}{\Gamma\left(\nu_{1}\right) \Gamma\left(\nu_{2}\right) \Gamma\left(D-\nu_{1}-\nu_{2}\right)},
$$

and the one-loop triangle integral with arbitrary powers and with one external mass scale $Q^{2}$ is given by (see for example [7])

$$
I_{3}^{D}\left(\nu_{1}, \nu_{2}, \nu_{3} ; Q^{2}\right)=(-1)^{\frac{D}{2}}\left(Q^{2}\right)^{\frac{D}{2}-\nu_{123}} \frac{\Gamma\left(\frac{D}{2}-\nu_{12}\right) \Gamma\left(\frac{D}{2}-\nu_{13}\right) \Gamma\left(\nu_{123}-\frac{D}{2}\right)}{\Gamma\left(\nu_{2}\right) \Gamma\left(\nu_{3}\right) \Gamma\left(D-\nu_{123}\right)},
$$

where we have introduced the shorthand $\nu_{i j}=\nu_{i}+\nu_{j}, \nu_{i j k}=\nu_{i}+\nu_{j}+\nu_{k}$, etc.

\section{The master topologies}

In this section, we collect the explicit expressions in terms of hypergeometric functions for the general master-topology integrals $A^{D}\left(B^{D}\right)$ and $C^{D}$, with arbitrary powers of propagators and general dimension $D$. We also show how to relate these to simpler pinched integrals and we compute explicitly the integrals that we need to evaluate all the tensor integrals in a closed analytical form and as an expansion in $\epsilon(D=4-2 \epsilon)$. 


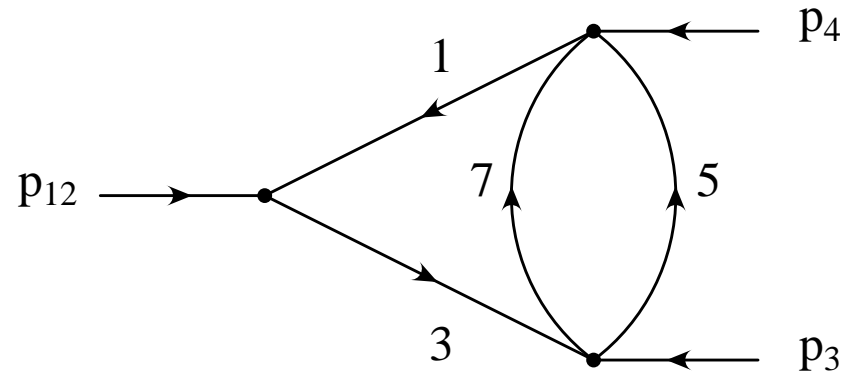

Figure 2: One-loop self-energy insertion into a one-loop triangle. The propagators are labelled according to Eq. (2.3) and are each raised to the $\nu_{i}$ power.

\subsection{Topology $A$}

The master-topology integral $A^{D}\left(\nu_{1}, \nu_{2}, \nu_{3}, \nu_{5}, \nu_{7} ; s, t\right)$ is shown in Fig. 3 and, with respect to the propagator factors of Eq. (2.3), is defined as

$$
A^{D}\left(\nu_{1}, \nu_{2}, \nu_{3}, \nu_{5}, \nu_{7} ; s, t\right)=\int \frac{d^{D} k_{1}}{i \pi^{D / 2}} \int \frac{d^{D} k_{2}}{i \pi^{D / 2}} \frac{1}{A_{1}^{\nu_{1}} A_{2}^{\nu_{2}} A_{3}^{\nu_{3}} A_{5}^{\nu_{5}} A_{7}^{\nu_{7}}} .
$$

This integral is related by a factor to the ordinary one-loop box integral

$$
A^{D}\left(\nu_{1}, \nu_{2}, \nu_{3}, \nu_{5}, \nu_{7} ; s, t\right)=\Pi^{D}\left(\nu_{5}, \nu_{7}\right) I_{4}^{D}\left(\nu_{1}, \nu_{2}, \nu_{3}, \nu_{5}+\nu_{7}-\frac{D}{2} ; s, t\right),
$$

where $\Pi^{D}\left(\nu_{5}, \nu_{7}\right)$ is given in Eq. (4.42) and

$$
I_{4}^{D}\left(\nu_{1}, \nu_{2}, \nu_{3}, \nu_{4} ; s, t\right)=\int \frac{d^{D} k_{1}}{i \pi^{D / 2}} \frac{1}{A_{1}^{\nu_{1}} A_{2}^{\nu_{2}} A_{3}^{\nu_{3}} A_{4}^{\nu_{4}}} .
$$

Expressions for the one-loop box integral with general powers of the propagators are easily obtained [4, 7, 8]. In the kinematic region $|t|<|s|$ we find [4]

$$
\begin{aligned}
& I_{4}^{D}\left(\nu_{1}, \nu_{2}, \nu_{3},\right.\left.\nu_{4} ; s, t\right)=(-1)^{\frac{D}{2}} s^{\frac{D}{2}-\nu_{1234}} \\
& \times \quad\left[\frac{\Gamma\left(\nu_{1234}-\frac{D}{2}\right) \Gamma\left(\frac{D}{2}-\nu_{234}\right) \Gamma\left(\frac{D}{2}-\nu_{124}\right)}{\Gamma\left(\nu_{1}\right) \Gamma\left(\nu_{3}\right) \Gamma\left(D-\nu_{1234}\right)}\right. \\
& \quad \times{ }_{3} F_{2}\left(\nu_{2}, \nu_{4}, \nu_{1234}-\frac{D}{2}, 1+\nu_{234}-\frac{D}{2}, 1+\nu_{124}-\frac{D}{2},-\frac{t}{s}\right) \\
&+\frac{\Gamma\left(\nu_{234}-\frac{D}{2}\right) \Gamma\left(\frac{D}{2}-\nu_{34}\right) \Gamma\left(\nu_{3}-\nu_{1}\right) \Gamma\left(\frac{D}{2}-\nu_{23}\right)}{\Gamma\left(\nu_{2}\right) \Gamma\left(\nu_{3}\right) \Gamma\left(\nu_{4}\right) \Gamma\left(D-\nu_{1234}\right)}
\end{aligned}
$$




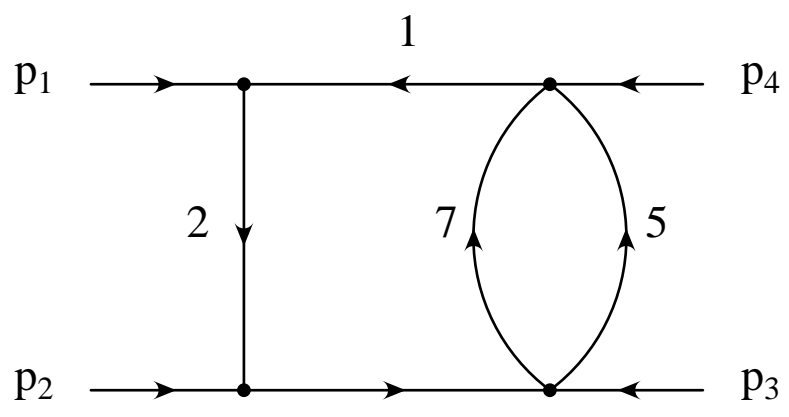

3

Figure 3: The master topology A. The propagators are labelled according to Eq. 2.3) and are each raised to the $\nu_{i}$ power.

$$
\begin{aligned}
& \times\left(\frac{t}{s}\right)^{\frac{D}{2}-\nu_{234}}{ }_{3} F_{2}\left(\nu_{1}, \frac{D}{2}-\nu_{34}, \frac{D}{2}-\nu_{23}, 1+\nu_{1}-\nu_{3}, 1+\frac{D}{2}-\nu_{234},-\frac{t}{s}\right) \\
+ & \frac{\Gamma\left(\nu_{124}-\frac{D}{2}\right) \Gamma\left(\frac{D}{2}-\nu_{14}\right) \Gamma\left(\nu_{1}-\nu_{3}\right) \Gamma\left(\frac{D}{2}-\nu_{12}\right)}{\Gamma\left(\nu_{1}\right) \Gamma\left(\nu_{2}\right) \Gamma\left(\nu_{4}\right) \Gamma\left(D-\nu_{1234}\right)} \\
& \left.\times\left(\frac{t}{s}\right)^{\frac{D}{2}-\nu_{124}}{ }_{3} F_{2}\left(\nu_{3}, \frac{D}{2}-\nu_{14}, \frac{D}{2}-\nu_{12}, 1+\nu_{3}-\nu_{1}, 1+\frac{D}{2}-\nu_{124},-\frac{t}{s}\right)\right] .
\end{aligned}
$$

Expressions for $I_{4}^{D}$ valid for $|s|<|t|$ can be obtained by the exchanges $\nu_{1} \leftrightarrow \nu_{2}, \nu_{3} \leftrightarrow \nu_{4}$ and $s \leftrightarrow t$ in Eq. (5.4).

Although these integrals appear with a variety of integer values for $\nu_{1}, \nu_{2}$ and $\nu_{3}$ in dimensions higher than $D=4-2 \epsilon$, we can systematically relate them to simpler integrals by the integration-by-parts identities. In fact, we find

$$
\begin{aligned}
s \nu_{1} \mathbf{1}^{+} I_{4}^{D} & =-\left(D-\nu_{12334}\right) I_{4}^{D}+\left(\nu_{1} \mathbf{1}^{+}+\nu_{2} \mathbf{2}^{+}+\nu_{4} \mathbf{4}^{+}\right) \mathbf{3}^{-} I_{4}^{D}, \\
\left(D-2-\nu_{1223}\right) \nu_{2} \mathbf{2}^{+} I_{4}^{D} & =\left(D-2-\nu_{1344}\right) \nu_{4} \mathbf{4}^{+} I_{4}^{D}+\left(\nu_{2}-\nu_{4}\right)\left(\nu_{1} \mathbf{1}^{+}+\nu_{3} \mathbf{3}^{+}\right) I_{4}^{D}, \\
s \nu_{3} \mathbf{3}^{+} I_{4}^{D} & =-\left(D-\nu_{11234}\right) I_{4}^{D}+\left(\nu_{2} \mathbf{2}^{+}+\nu_{3} \mathbf{3}^{+}+\nu_{4} \mathbf{4}^{+}\right) \mathbf{1}^{-} I_{4}^{D},
\end{aligned}
$$

where we have used the shorthand notation $I_{4}^{D}=I_{4}^{D}\left(\nu_{1}, \nu_{2}, \nu_{3}, \nu_{4} ; s, t\right)$ and $\nu_{i j j k}=\nu_{i}+2 \nu_{j}+\nu_{k}$, etc.

Repeated application of these identities reduces $\nu_{1}, \nu_{2}$ and $\nu_{3}$ to unity together with integrals where one of the propagators is pinched out, yielding a one-loop triangle (see Eq. (4.43))

$$
\begin{aligned}
& I_{4}^{D}\left(\nu_{1}, \nu_{2}, 0, \nu_{4} ; s, t\right)=I_{3}^{D}\left(\nu_{1}, \nu_{2}, \nu_{4} ; t\right) \\
& I_{4}^{D}\left(0, \nu_{2}, \nu_{3}, \nu_{4} ; s, t\right)=I_{3}^{D}\left(\nu_{3}, \nu_{2}, \nu_{4} ; t\right) .
\end{aligned}
$$


Subsequent application of

$$
t \nu_{4} \mathbf{4}^{+} I_{4}^{D}=-\left(D-\nu_{12234}\right) I_{4}^{D}+\left(\nu_{1} \mathbf{1}^{+}+\nu_{3} 3^{+}+\nu_{4} \mathbf{4}^{+}\right) \mathbf{2}^{-} I_{4}^{D},
$$

can be used to control the power of $\nu_{4}$ and form the pinched triangle integral

$$
I_{4}^{D}\left(\nu_{1}, 0, \nu_{3}, \nu_{4} ; s, t\right)=I_{3}^{D}\left(\nu_{4}, \nu_{1}, \nu_{3} ; s\right) .
$$

Equation (5.10) should be used until $\nu_{4}=2-D / 2$, corresponding to $\nu_{5}=\nu_{7}=1$, that is the master integral for the $A$ topology.

The dimensional-shift identity for the master integral is obtained applying

$$
\mathbf{d}^{-}=\left[\left(\nu_{3} \mathbf{3}^{+}+\nu_{2} \mathbf{2}^{+}+\nu_{1} \mathbf{1}^{+}\right)\left(\nu_{5} \mathbf{5}^{+}+\nu_{7} \mathbf{7}^{+}\right)+\nu_{5} 5^{+} \nu_{7} 7^{+}\right]
$$

to $A^{D+2}(1,1,1,1,1 ; s, t)$, and reducing the right-hand side with the help of Eqs. (5.5)-(5.7) and $(5.10)$ :

$$
\begin{aligned}
A^{D+2}(1,1,1,1,1 ; s, t) & =\frac{(D-4) s t^{2}}{3(D-1)(3 D-10)(3 D-8)(t+s)} A^{D}(1,1,1,1,1 ; s, t) \\
& +\frac{s[(D-4) t+(2 D-6) s]}{3(D-2)(D-1)(3 D-8)(t+s)} I_{3}^{D}\left(2-\frac{D}{2}, 1,1 ; s\right) \Pi^{D}(1,1) \\
& +\frac{t}{3(D-4)(D-1)(t+s)} I_{2}^{D}\left(2-\frac{D}{2}, 1 ; t\right) \Pi^{D}(1,1)
\end{aligned}
$$

where

$$
I_{2}^{D}\left(\nu_{1}, \nu_{2} ; Q^{2}\right)=\left(Q^{2}\right)^{\frac{D}{2}-\nu_{12}} \Pi^{D}\left(\nu_{1}, \nu_{2}\right) .
$$

The master integral for the $A$ topology has all propagators raised to unit powers and may written in terms of Gaussian hypergeometric functions as [4]

$$
\begin{aligned}
A^{D}(1,1,1,1,1 ; s, t)= & -\frac{\Gamma^{2}\left(2-\frac{D}{2}\right) \Gamma^{2}\left(\frac{D}{2}-1\right) \Gamma(4-D) \Gamma(D-3)}{\Gamma\left(\frac{3 D}{2}-5\right) \Gamma(D-2) \Gamma\left(3-\frac{D}{2}\right)} \\
\times & {\left[(-t)^{D-5} \frac{\Gamma\left(\frac{D}{2}-2\right)}{\Gamma\left(2-\frac{D}{2}\right)}{ }_{2} F_{1}\left(1,1, \frac{D}{2}-1, \frac{s+t}{t}\right)\right.} \\
& \left.+(-s)^{D-5} \Gamma(D-3){ }_{2} F_{1}\left(1,2-\frac{D}{2}, \frac{D}{2}-1, \frac{s+t}{s}\right)\right] .
\end{aligned}
$$

Making the expansion in $\epsilon=D / 2-2$ of the hypergeometric functions, we obtain

$$
A^{D}(1,1,1,1,1 ; s, t)=\frac{\Gamma^{3}(1-\epsilon) \Gamma(1+2 \epsilon)}{2 s(1-2 \epsilon) \epsilon^{3} \Gamma(1-3 \epsilon)}\left[(-s)^{-2 \epsilon} A_{1}(s, t)+(-t)^{-2 \epsilon} A_{2}(s, t)\right]
$$

where $A_{1}(s, t)$ and $A_{2}(s, t)$ are given respectively by: 
1) in the physical region $s>0, t<0$ :

$$
\begin{aligned}
A_{1}(s, t)= & \left(-\frac{t}{s}\right)^{-\epsilon}\left\{1-\epsilon^{2}\left[\operatorname{Li}_{2}\left(\frac{s+t}{t}\right)-2 \zeta_{2}\right]-\epsilon^{3}\left[\operatorname{Li}_{3}\left(\frac{s+t}{t}\right)+\mathrm{S}_{1,2}\left(\frac{s+t}{t}\right)\right.\right. \\
& \left.-2 \zeta_{3}\right]-\epsilon^{4}\left[\operatorname{Li}_{4}\left(\frac{s+t}{t}\right)+\mathrm{S}_{2,2}\left(\frac{s+t}{t}\right)+\mathrm{S}_{1,3}\left(\frac{s+t}{t}\right)\right. \\
& \left.\left.+2 \zeta_{2} \operatorname{Li}_{2}\left(\frac{s+t}{t}\right)-9 \zeta_{4}\right]\right\}+\mathcal{O}\left(\epsilon^{5}\right) \\
A_{2}(s, t)= & +\epsilon \log \left(-\frac{t}{s}\right)-\epsilon^{2} \operatorname{Li}_{2}\left(\frac{s+t}{s}\right)-\epsilon^{3} \operatorname{Li}_{3}\left(\frac{s+t}{s}\right)-\epsilon^{4} \operatorname{Li}_{4}\left(\frac{s+t}{s}\right) \\
& +\mathcal{O}\left(\epsilon^{5}\right),
\end{aligned}
$$

2) while in the region $s<0, t<0$ :

$$
\begin{aligned}
A_{1}(s, t)= & \left(\frac{t}{s}\right)^{-\epsilon}\left\{1+\epsilon^{2}\left[\operatorname{Li}_{2}\left(\frac{t}{s+t}\right)+\frac{1}{2} \log ^{2}\left(\frac{s+t}{t}\right)-\frac{\pi^{2}}{2}\right]-\epsilon^{3}\left[2 \operatorname{Li}_{3}\left(\frac{t}{s+t}\right)\right.\right. \\
& \left.-\mathrm{S}_{1,2}\left(\frac{t}{s+t}\right)-\zeta_{3}+\log \left(\frac{s+t}{t}\right)\left(\operatorname{Li}_{2}\left(\frac{t}{s+t}\right)+\frac{5}{6} \pi^{2}\right)\right] \\
& +\epsilon^{4}\left[\mathrm{~S}_{1,3}\left(\frac{t}{s+t}\right)-2 \mathrm{~S}_{2,2}\left(\frac{t}{s+t}\right)+4 \operatorname{Li}_{4}\left(\frac{t}{s+t}\right)-\frac{23}{180} \pi^{4}\right. \\
& \left.+\frac{1}{24} \log ^{4}\left(\frac{s+t}{t}\right)+\log \left(\frac{s+t}{t}\right)\left(2 \operatorname{Li}_{3}\left(\frac{t}{s+t}\right)-\mathrm{S}_{1,2}\left(\frac{t}{s+t}\right)-\zeta_{3}\right)\right] \\
& \left.+\frac{\pi^{2}}{3} \operatorname{Li}_{2}\left(\frac{t}{s+t}\right)-\frac{\pi^{2}}{4} \log { }^{2}\left(\frac{s+t}{t}\right)+\frac{1}{2} \log ^{2}\left(\frac{s+t}{t}\right) \operatorname{Li}_{2}\left(\frac{t}{s+t}\right)\right\} \\
& +\mathcal{O}\left(\epsilon^{5}\right), \\
& +\epsilon \log \left(\frac{t}{s}\right)+\epsilon^{2}\left[\operatorname{Li}_{2}\left(\frac{s}{s+t}\right)+\frac{1}{2} \log ^{2}\left(\frac{s+t}{s}\right)-\frac{\pi^{2}}{3}\right] \\
A_{2}(s, t)= & \epsilon^{3}\left[\operatorname{Li}_{3}\left(\frac{s}{s+t}\right)+\frac{\pi^{2}}{3} \log \left(\frac{s+t}{s}\right)-\frac{1}{6} \log ^{3}\left(\frac{s+t}{s}\right)\right] \\
& +\epsilon^{4}\left[\operatorname{Li}_{4}\left(\frac{s}{s+t}\right)-\frac{\pi^{4}}{45}-\frac{\pi^{2}}{6} \log ^{2}\left(\frac{s+t}{s}\right)+\frac{1}{24} \log ^{4}\left(\frac{s+t}{s}\right)\right] \\
+ & \mathcal{O}\left(\epsilon^{5}\right) \cdot
\end{aligned}
$$




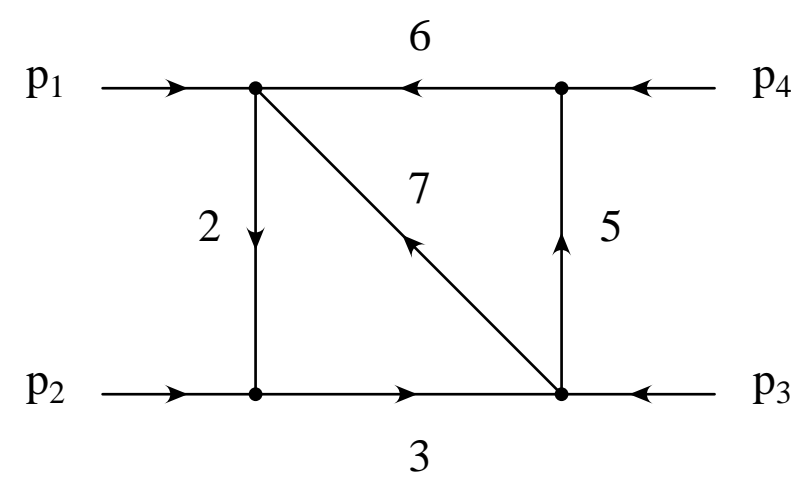

Figure 4: The master topology $C$. The propagators are labelled according to Eq. (2.3) and are each raised to the $\nu_{i}$ power.

\subsection{Topology $C$}

The diagonal-box master-topology integral is shown in Fig. 1 and with respect to the propagator factors of Eq. (2.3) is defined as

$$
C^{D}\left(\nu_{2}, \nu_{3}, \nu_{5}, \nu_{6}, \nu_{7} ; s, t\right)=\int \frac{d^{D} k_{1}}{i \pi^{D / 2}} \int \frac{d^{D} k_{2}}{i \pi^{D / 2}} \frac{1}{A_{2}^{\nu_{2}} A_{3}^{\nu_{3}} A_{5}^{\nu_{5}} A_{6}^{\nu_{6}} A_{7}^{\nu_{7}}} .
$$

For this graph, it is easy to read off the values of $\mathcal{Q}$ and $\mathcal{P}$ of Eqs. (4.8) and (4.10)

$$
\begin{aligned}
& \mathcal{Q}=x_{3} x_{6} x_{7} s+x_{2} x_{5} x_{7} t \\
& \mathcal{P}=x_{7}\left(x_{2}+x_{3}+x_{5}+x_{6}\right)+\left(x_{2}+x_{3}\right)\left(x_{5}+x_{6}\right) .
\end{aligned}
$$

The integral can be written as a single Mellin-Barnes integral (see for example Refs. [2, 3])

$$
\begin{aligned}
& C^{D}\left(\nu_{2}, \nu_{3}, \nu_{5}, \nu_{6}, \nu_{7} ; s, t\right)=\frac{(-1)^{D} s^{D-\nu_{23567}}}{\Gamma\left(\nu_{2}\right) \Gamma\left(\nu_{3}\right) \Gamma\left(\nu_{5}\right) \Gamma\left(\nu_{6}\right) \Gamma\left(\nu_{7}\right)} \\
& \quad \times \frac{\Gamma\left(\frac{D}{2}-\nu_{7}\right) \Gamma\left(\frac{D}{2}-\nu_{56}\right) \Gamma\left(\frac{D}{2}-\nu_{23}\right)}{\Gamma\left(D-\nu_{237}\right) \Gamma\left(D-\nu_{567}\right) \Gamma\left(\frac{3}{2} D-\nu_{23567}\right)} \int_{-i \infty}^{i \infty} \frac{d \alpha}{2 \pi i} \Gamma(-\alpha) \Gamma\left(D+\nu_{2}-\nu_{23567}-\alpha\right) \\
& \quad \times \Gamma\left(D+\nu_{5}-\nu_{23567}-\alpha\right) \Gamma\left(\nu_{23567}-D+\alpha\right) \Gamma\left(\nu_{3}+\alpha\right) \Gamma\left(\nu_{6}+\alpha\right)\left(\frac{t}{s}\right)^{\alpha},
\end{aligned}
$$

where the path of integration over $\alpha$ must be chosen so that to separate the poles coming from $\Gamma(\ldots-\alpha)$ from those coming from $\Gamma(\ldots+\alpha)$. In the kinematic region $|t|<|s|$ the contour at the infinity must be closed to the right, and the expression we obtain is

$$
C^{D}\left(\nu_{2}, \nu_{3}, \nu_{5}, \nu_{6}, \nu_{7} ; s, t\right)=(-1)^{D} s^{D-\nu_{23567}} \frac{\Gamma\left(\frac{D}{2}-\nu_{23}\right) \Gamma\left(\frac{D}{2}-\nu_{56}\right) \Gamma\left(\frac{D}{2}-\nu_{7}\right)}{\Gamma\left(\nu_{7}\right) \Gamma\left(\frac{3}{2} D-\nu_{23567}\right)}
$$




$$
\begin{aligned}
\times \quad & \frac{\Gamma\left(\nu_{23567}-D\right) \Gamma\left(D-\nu_{2367}\right) \Gamma\left(D-\nu_{3567}\right)}{\Gamma\left(\nu_{2}\right) \Gamma\left(\nu_{5}\right) \Gamma\left(D-\nu_{567}\right) \Gamma\left(D-\nu_{237}\right)} \\
& \times{ }_{3} F_{2}\left(\nu_{3}, \nu_{6}, \nu_{23567}-D, 1-D+\nu_{2367}, 1-D+\nu_{3567},-\frac{t}{s}\right) \\
+ & \frac{\Gamma\left(\nu_{2}-\nu_{5}\right) \Gamma\left(D-\nu_{267}\right) \Gamma\left(\nu_{2367}-D\right)}{\Gamma\left(\nu_{2}\right) \Gamma\left(\nu_{3}\right) \Gamma\left(\nu_{6}\right) \Gamma\left(D-\nu_{567}\right)} \\
& \times\left(\frac{t}{s}\right)^{D-\nu_{2367}}{ }_{3} F_{2}\left(\nu_{5}, D-\nu_{267}, D-\nu_{237}, 1+D-\nu_{2367}, 1+\nu_{5}-\nu_{2},-\frac{t}{s}\right) \\
+ & \frac{\Gamma\left(\nu_{5}-\nu_{2}\right) \Gamma\left(D-\nu_{357}\right) \Gamma\left(\nu_{3567}-D\right)}{\Gamma\left(\nu_{3}\right) \Gamma\left(\nu_{5}\right) \Gamma\left(\nu_{6}\right) \Gamma\left(D-\nu_{237}\right)} \\
& \left.\times\left(\frac{t}{s}\right)^{D-\nu_{3567}}{ }_{3} F_{2}\left(\nu_{2}, D-\nu_{357}, D-\nu_{567}, 1+D-\nu_{3567}, 1+\nu_{2}-\nu_{5},-\frac{t}{s}\right)\right] .
\end{aligned}
$$

The solution valid when $|s|<|t|$ can be obtained from Eq. (5.25) by the exchanges

$$
s \leftrightarrow t, \quad \nu_{2} \leftrightarrow \nu_{3}, \quad \nu_{5} \leftrightarrow \nu_{6}
$$

The expression for the diagonal box (5.25) has an apparent singularity when $\nu_{2}-\nu_{5}$ is an integer which cancels in the actual evaluation of the diagram.

In addition, although in principle we need to know this integral for arbitrary powers and arbitrary dimension, further simplifications occur. Integration by parts yields the following relations $\left(C^{D}=C^{D}\left(\nu_{2}, \nu_{3}, \nu_{5}, \nu_{6}, \nu_{7} ; s, t\right)\right)$

$$
\begin{aligned}
& \left(D-2-2 \nu_{23}\right) \nu_{2} \mathbf{2}^{+} C^{D}=\left(D-2-2 \nu_{7}\right) \nu_{7} 7^{+} C^{D}-\left(D-2-2 \nu_{23}\right) \nu_{3} 3^{+} C^{D} \\
& \left(D-2-2 \nu_{56}\right) \nu_{6} \mathbf{6}^{+} C^{D}=\left(D-2-2 \nu_{7}\right) \nu_{7} 7^{+} C^{D}-\left(D-2-2 \nu_{56}\right) \nu_{5} 5^{+} C^{D}
\end{aligned}
$$

so that we can reduce both $\nu_{2}$ and $\nu_{6}$ to unity at the expense of increasing $\nu_{3}$ and $\nu_{5}$ together with $\nu_{7}$. We now reduce $\nu_{3}$ and $\nu_{5}$ to unity using the relations

$$
\begin{aligned}
s\left(D-2-2 \nu_{23}\right) \nu_{3} \mathbf{3}^{+} C^{D=} & -\left(D-1-\nu_{237}\right)\left(3 D-2 \nu_{235667}\right) C^{D} \\
& +2\left(D-1-\nu_{237}\right) \nu_{5} \mathbf{5}^{+} \mathbf{6}^{-} C^{D}+\left(D-2-2 \nu_{7}\right) \nu_{7} \mathbf{7}^{+} \mathbf{6}^{-} C^{D}, \\
t\left(D-2-2 \nu_{56}\right) \nu_{5} \mathbf{5}^{+} C^{D=} & -\left(D-1-\nu_{567}\right)\left(3 D-2 \nu_{223567}\right) C^{D} \\
& +2\left(D-1-\nu_{567}\right) \nu_{3} \mathbf{3}^{+} \mathbf{2}^{-} C^{D}+\left(D-2-2 \nu_{7}\right) \nu_{7} \mathbf{7}^{+} \mathbf{2}^{-} C^{D},
\end{aligned}
$$

which, because $\nu_{2}$ and $\nu_{6}$ are already unity, produces simpler pinched integrals of the form

$$
\begin{aligned}
& C^{D}\left(0, \nu_{3}, \nu_{5}, \nu_{6}, \nu_{7} ; s, t\right)=\Pi^{D}\left(\nu_{3}, \nu_{7}\right) I_{3}^{D}\left(\nu_{5}, \nu_{6}, \nu_{3}+\nu_{7}-\frac{D}{2} ; s\right) \\
& C^{D}\left(\nu_{2}, \nu_{3}, \nu_{5}, 0, \nu_{7} ; s, t\right)=\Pi^{D}\left(\nu_{5}, \nu_{7}\right) I_{3}^{D}\left(\nu_{3}, \nu_{2}, \nu_{5}+\nu_{7}-\frac{D}{2} ; t\right)
\end{aligned}
$$


where the self-energy factor $\Pi^{D}$ is defined in Eq. (4.42) and the one-loop triangle $I_{3}^{D}$ is given by Eq. (4.43).

When the outer propagators have unit powers, we can reduce $\nu_{7}$ using

$$
\begin{aligned}
s t\left(D-2-2 \nu_{7}\right) \nu_{7} \mathbf{7}^{+} C^{D}= & -(s+t)\left(D-3-\nu_{7}\right)\left(3 D-10-2 \nu_{7}\right) C^{D} \\
& +2\left(D-3-\nu_{7}\right)\left(t \mathbf{5}^{+} \mathbf{6}^{-}+s \mathbf{6}^{+} \mathbf{5}^{-}\right) C^{D} \\
& +\left(D-2-2 \nu_{7}\right)\left(t \nu_{7} \mathbf{7}^{+} \mathbf{6}^{-}+s \nu_{7} \mathbf{7}^{+} \mathbf{5}^{-}\right) C^{D} .
\end{aligned}
$$

This equation is only valid when $\nu_{2}=\nu_{3}=\nu_{5}=\nu_{6}=1$.

Finally, applying

$$
\mathbf{d}^{-}=\nu_{7} 7^{+}\left(\nu_{2} \mathbf{2}^{+}+\nu_{3} 3^{+}+\nu_{5} 5^{+}+\nu_{6} \mathbf{6}^{+}\right)+\left(\nu_{2} \mathbf{2}^{+}+\nu_{3} 3^{+}\right)\left(\nu_{5} 5^{+}+\nu_{6} \mathbf{6}^{+}\right)
$$

to $C^{D+2}(1,1,1,1,1 ; s, t)$, we can obtain the dimensional-shift equation

$$
\begin{aligned}
C^{D+2}(1,1,1,1,1 ; s, t)= & \frac{(D-4)^{2} s^{2} t^{2}}{3(D-3)(D-2)(3 D-10)(3 D-8)(t+s)^{2}} C^{D}(1,1,1,1,1 ; s, t) \\
& +\frac{s[(2 D-5) t+(D-3) s]}{3(D-3)(D-2)^{2}(t+s)^{2}} I_{2}^{D}\left(2-\frac{D}{2}, 1 ; s\right) \Pi^{D}(1,1) \\
& +\frac{t[(D-3) t+(2 D-5) s]}{3(D-3)(D-2)^{2}(t+s)^{2}} I_{2}^{D}\left(2-\frac{D}{2}, 1 ; t\right) \Pi^{D}(1,1) . \quad(5.34)
\end{aligned}
$$

To summarize, we actually only need to know $C^{D}(1,1,1,1,1 ; s, t)$ rather than the more general case. With the propagator powers equal to unity, all of the ${ }_{3} F_{2}$ functions of Eq. (5.25) reduce to ${ }_{2} F_{1}$. To deal with the pole in $\left(\nu_{2}-\nu_{5}\right)$ we proceed as in Ref. [7]: we set $\nu_{2}=\nu_{5}+\delta$, and, after performing an appropriate analytical continuation, we take the limit $\delta \rightarrow 0$. The final expression is given by

$$
\begin{aligned}
C^{D}(1,1,1,1,1 ; s, t)= & -\frac{\Gamma\left(\frac{D}{2}-1\right) \Gamma(3-D) \Gamma^{2}\left(\frac{D}{2}-2\right)}{\Gamma\left(\frac{3}{2} D-5\right)} \\
\times & {\left[(-t)^{D-5}{ }_{2} F_{1}\left(1,1, D-2, \frac{s+t}{t}\right)\right.} \\
& \left.+(-s)^{D-5}{ }_{2} F_{1}\left(1,1, D-2, \frac{s+t}{s}\right)\right] .
\end{aligned}
$$

If we make a series expansion in $\epsilon$, we obtain

$$
C^{D}(1,1,1,1,1 ; s, t)=\frac{\Gamma^{3}(1-\epsilon) \Gamma(1+2 \epsilon)}{2(s+t) \Gamma(1-3 \epsilon) \epsilon^{3}}\left[(-s)^{-2 \epsilon} C(s, t)+(-t)^{-2 \epsilon} C(t, s)\right]
$$

where $C(s, t)$ is given respectively by: 
1) in the physical region $s>0, t<0$ :

$$
\begin{aligned}
C(s, t)= & \log \left(-\frac{t}{s}\right)+2 \epsilon \operatorname{Li}_{2}\left(\frac{s+t}{t}\right)+4 \epsilon^{2} \operatorname{Li}_{3}\left(\frac{s+t}{t}\right)+8 \epsilon^{3} \operatorname{Li}_{4}\left(\frac{s+t}{t}\right) \\
& +\mathcal{O}\left(\epsilon^{4}\right),
\end{aligned}
$$

2) while in the region $s<0, t<0$ :

$$
\begin{aligned}
C(s, t)= & \log \left(\frac{t}{s}\right)-2 \epsilon\left[\operatorname{Li}_{2}\left(\frac{t}{s+t}\right)+\frac{1}{2} \log ^{2}\left(\frac{s+t}{t}\right)-\frac{\pi^{2}}{3}\right] \\
& +4 \epsilon^{2}\left[\operatorname{Li}_{3}\left(\frac{t}{s+t}\right)-\frac{1}{6} \log ^{3}\left(\frac{s+t}{t}\right)+\frac{\pi^{2}}{3} \log \left(\frac{s+t}{t}\right)\right] \\
& -8 \epsilon^{3}\left[\operatorname{Li}_{4}\left(\frac{t}{s+t}\right)+\frac{1}{24} \log ^{4}\left(\frac{s+t}{t}\right)-\frac{\pi^{2}}{6} \log ^{2}\left(\frac{s+t}{t}\right)-\frac{\pi^{4}}{45}\right] \\
& +\mathcal{O}\left(\epsilon^{4}\right) .
\end{aligned}
$$

Note that the prefactor of Eq. (5.35) indicates that the integral diverges as $1 / \epsilon^{3}$. However, the hypergeometric functions conspire to remove the leading divergence and we reproduce the result for $C^{D}(1,1,1,1,1 ; s, t)$ quoted in Ref. [2].

\section{Conclusions}

Finally we summarize what we have accomplished in this paper. The pentabox with lightlike external legs is one of the two-loop box graphs needed for the evaluation of Feynman diagrams for physical $2 \rightarrow 2$ scattering processes, the others being the recently evaluated planar double-box graph [1, 2] and non-planar double-box graph [3] as well as one-loop box integrals with bubble insertions on one of the propagators [4]. Using integration by parts identities, we have derived explicit analytic expressions for the scalar pentabox graph $J^{D}(1,1,1,1,1,1,1 ; s, t)$ in terms of two simpler box integrals $(A$ and $C)$ with fewer propagators and given an explicit expansion in $\epsilon$ in terms of polylogarithm functions (see Eq. (3.13)).

In Section twe demonstrated how generic two-loop integrals could be expressed directly in terms of integrals in higher dimension with shifted propagator powers. Similar results have been given by Tarasov [6] using differential operators; however we believe our method based on completing the square and Gaussian integration to be more direct and simpler to apply to generic integrals where the quantities $a, b, c, d^{\mu}, e^{\mu}$ and $f$ (see Eq. (4.3)) can easily be read off from the graph and used to construct the vectors $\mathcal{X}^{\mu}$ and $\mathcal{Y}^{\nu}$ (defined in Eq. (4.7)), which therefore are linear in both the external momenta and the $x_{i}$. As detailed in Section $⿴$, the powers of $x_{i}$ and $\mathcal{P}$ present in Eqs. (4.22) and (4.25)-(4.37) can then be straightforwardly exchanged for scalar integrals with higher $\nu_{i}$ and higher $D$. 
In the case of the pentabox, the tensor integrals can also be written in terms of the same master-topology integrals as the scalar pentabox, but with different powers of propagators and in higher dimension. We give quite general analytic formulae for the master-topology integrals with arbitrary dimension and propagators. For the $A$ topology we quote the results of [4] while for the $C$ topology we employ the Mellin-Barnes integral representation. Other methods would also suffice. In fact, it is not necessary to know the master integrals in their full generality since further integration-by-parts identities relate the various integrals. Explicit expressions for topology $A$ and $C$ are given in Eqs. (5.15) and (5.35) respectively, together with instructions on how to apply them to more general integrals.

Finally, we note that the recurrence relations necessary for the complete reduction of tensor integrals for the planar-box graph were described in Ref. [2]. However, the situation for the non-planar box is less well developed. At present only one master integral is known, and the solution of the recurrence relations for that topology is an open problem.

\section{Acknowledgements}

We thank J.V. Armitage, J.B. Tausk, M.E. Tejeda-Yeomans, J.J. van der Bij and M. Zimmer for assistance and useful suggestions and D. Broadhurst for his encouragement. C.A. acknowledges the financial support of the Greek Government and C.O. acknowledges the financial support of the INFN. We gratefully acknowledge the support of the British Council and German Academic Exchange Service under ARC project 1050.

\section{A Polylogarithms}

The purpose of this appendix is to define the generalised polylogarithms that occur in the expansion in $\epsilon$ of the pentabox scalar and tensor loop integrals and to give useful identities amongst the polylogarithms. In Section A.1 we give the definitions of the polylogarithm functions $\mathrm{S}_{n, p}(x)$. These functions are real when $x \leq 1$ but they develop an imaginary part for $x>1$. Analytic continuation formulae are given in Section A.2. Finally, useful identities between polylogarithms are listed in Section A.3.

\section{A.1 Definition}

The generalised polylogarithms of Nielsen are defined by

$$
\mathrm{S}_{n, p}(x)=\frac{(-1)^{n+p-1}}{(n-1) ! p !} \int_{0}^{1} d t \frac{\log ^{n-1}(t) \log ^{p}(1-x t)}{t}, \quad n, p \geq 1, \quad x \leq 1 .
$$


For $p=1$ we find the usual polylogarithms

$$
\mathrm{S}_{n-1,1}(x) \equiv \operatorname{Li}_{n}(x)
$$

The $S_{n, p}$ 's with argument $x, 1-x$ and $1 / x$ can be related to each other via [9]

$$
\begin{aligned}
\mathrm{S}_{n, p}(1-x) & =\sum_{s=0}^{n-1} \frac{\log ^{s}(1-x)}{s !}\left[\mathrm{S}_{n-s, p}(1)-\sum_{r=0}^{p-1} \frac{(-1)^{r}}{r !} \log ^{r}(x) \mathrm{S}_{p-r, n-s}(x)\right] \\
& +\frac{(-1)^{p}}{n ! p !} \log ^{n}(1-x) \log ^{p}(x), \\
\mathrm{S}_{n, p}\left(\frac{1}{x}\right) & =(-1)^{n} \sum_{s=0}^{p-1}(-1)^{s} \sum_{r=0}^{s} \frac{(-1)^{r}}{r !} \log ^{r}(-x)\left(\begin{array}{c}
n+s-r-1 \\
s-r
\end{array}\right) \mathrm{S}_{n+s-r, p-s}(x) \\
& +\sum_{r=0}^{n-1} \frac{(-1)^{r+p}}{r !} \log ^{r}(-x) C_{n-r, p}+\frac{(-1)^{n}}{(n+p) !} \log ^{n+p}(-x)
\end{aligned}
$$

with

$$
\begin{aligned}
C_{n, p} & =(-1)^{n+1} \sum_{r=1}^{p-1}(-1)^{p-r}\left(\begin{array}{c}
n+r-1 \\
r
\end{array}\right) \mathrm{S}_{n+r, p-r}(-1) \\
& +(-1)^{p}\left(1-(-1)^{n}\right) \mathrm{S}_{n, p}(-1) .
\end{aligned}
$$

The usual binomial coefficient is defined as

$$
\left(\begin{array}{l}
a \\
b
\end{array}\right)=\frac{a !}{b !(a-b) !} .
$$

We also need the definition of the Riemann Zeta functions

$$
\zeta_{n}=\sum_{s=1}^{\infty} \frac{1}{s^{n}},
$$

and in particular

$$
\zeta_{2}=\frac{\pi^{2}}{6}, \quad \zeta_{3}=1.20206 \ldots \quad \zeta_{4}=\frac{\pi^{4}}{90}
$$

\section{A.2 Analytic continuation formulae}

For $x>1$, the following analytic continuations should be used

$$
\begin{aligned}
& \operatorname{Li}_{2}(x+i 0)=-\mathrm{Li}_{2}\left(\frac{1}{x}\right)-\frac{1}{2} \log ^{2}(x)+\frac{\pi^{2}}{3}+i \pi \log (x) \\
& \operatorname{Li}_{3}(x+i 0)=\operatorname{Li}_{3}\left(\frac{1}{x}\right)-\frac{1}{6} \log ^{3}(x)+\frac{\pi^{2}}{3} \log (x)+\frac{i \pi}{2} \log ^{2}(x)
\end{aligned}
$$




$$
\begin{aligned}
\mathrm{Li}_{4}(x+i 0)= & -\mathrm{Li}_{4}\left(\frac{1}{x}\right)-\frac{1}{24} \log ^{4}(x)+\frac{\pi^{2}}{6} \log ^{2}(x)+\frac{\pi^{4}}{45}+\frac{i \pi}{6} \log ^{3}(x) \\
\mathrm{S}_{1,2}(x+i 0)= & -\mathrm{S}_{1,2}\left(\frac{1}{x}\right)+\mathrm{Li}_{3}\left(\frac{1}{x}\right)+\log (x) \mathrm{Li}_{2}\left(\frac{1}{x}\right)+\frac{1}{6} \log ^{3}(x)-\frac{\pi^{2}}{2} \log (x)+\zeta_{3} \\
& +i \pi\left[\frac{\pi^{2}}{6}-\mathrm{Li}_{2}\left(\frac{1}{x}\right)-\frac{1}{2} \log ^{2}(x)\right] \\
\mathrm{S}_{1,3}(x+i 0)= & -\mathrm{S}_{1,3}\left(\frac{1}{x}\right)+\mathrm{S}_{2,2}\left(\frac{1}{x}\right)+\log (x) \mathrm{S}_{1,2}\left(\frac{1}{x}\right)-\operatorname{Li}_{4}\left(\frac{1}{x}\right)-\log (x) \operatorname{Li}_{3}\left(\frac{1}{x}\right) \\
& -\frac{1}{2} \log ^{2}(x) \operatorname{Li}_{2}\left(\frac{1}{x}\right)+\frac{\pi^{2}}{2} \operatorname{Li}\left(\frac{1}{x}\right)-\frac{1}{24} \log ^{4}(x)+\frac{\pi^{2}}{4} \log ^{2}(x)-\frac{19 \pi^{4}}{360} \\
& +i \pi\left[\operatorname{Li}_{3}\left(\frac{1}{x}\right)-\mathrm{S}_{1,2}\left(\frac{1}{x}\right)+\log (x) \operatorname{Li}_{2}\left(\frac{1}{x}\right)+\frac{1}{6} \log ^{3}(x)-\frac{\pi^{2}}{6} \log (x)\right]
\end{aligned}
$$

$$
\begin{aligned}
\mathrm{S}_{2,2}(x+i 0)= & \mathrm{S}_{2,2}\left(\frac{1}{x}\right)-2 \operatorname{Li}_{4}\left(\frac{1}{x}\right)-\log (x) \operatorname{Li}_{3}\left(\frac{1}{x}\right)+\frac{1}{24} \log ^{4}(x)-\frac{\pi^{2}}{4} \log ^{2}(x) \\
& +\zeta_{3} \log (x)+\frac{\pi^{4}}{45}+i \pi\left[\operatorname{Li}_{3}\left(\frac{1}{x}\right)-\frac{1}{6} \log ^{3}(x)+\frac{\pi^{2}}{6} \log (x)-\zeta_{3}\right]
\end{aligned}
$$

\section{A.3 Useful identities}

For the expansions of hypergeometric functions given in this paper, the argument of the polylogarithms is always either $(s+t) / s$ or $(s+t) / t$, which are related by the following identities

$$
\begin{aligned}
\mathrm{Li}_{2}\left(\frac{x}{x-1}\right) & =-\mathrm{Li}_{2}(x)-\frac{1}{2} \log ^{2}(1-x), \\
\mathrm{Li}_{3}\left(\frac{x}{x-1}\right)= & -\mathrm{Li}_{3}(x)+\mathrm{S}_{1,2}(x)+\log (1-x) \operatorname{Li}_{2}(x)+\frac{1}{6} \log ^{3}(1-x), \\
\mathrm{Li}_{4}\left(\frac{x}{x-1}\right)= & -\mathrm{Li}_{4}(x)+\mathrm{S}_{2,2}(x)-\mathrm{S}_{1,3}(x)+\log (1-x) \operatorname{Li}_{3}(x)-\log (1-x) \mathrm{S}_{1,2}(x) \\
& -\frac{1}{2} \log ^{2}(1-x) \operatorname{Li}_{2}(x)-\frac{1}{24} \log ^{4}(1-x), \\
\mathrm{S}_{1,2}\left(\frac{x}{x-1}\right)= & \mathrm{S}_{1,2}(x)-\frac{1}{6} \log ^{3}(1-x), \\
\mathrm{S}_{1,3}\left(\frac{x}{x-1}\right)= & -\mathrm{S}_{1,3}(x)-\frac{1}{24} \log ^{4}(1-x), \\
\mathrm{S}_{2,2}\left(\frac{x}{x-1}\right)= & \mathrm{S}_{2,2}(x)-2 \mathrm{~S}_{1,3}(x)-\log (1-x) \mathrm{S}_{1,2}(x)+\frac{1}{24} \log ^{4}(1-x) .
\end{aligned}
$$




\section{References}

[1] V.A. Smirnov, Phys. Lett. B460 (99) 397.

[2] V.A. Smirnov and O.L. Veretin, Nucl. Phys. B566 (2000) 469-485.

[3] J.B. Tausk, Phys. Lett. B469 (1999) 225.

[4] C. Anastasiou, E.W.N. Glover and C. Oleari, Nucl. Phys. B565 (2000) 445.

[5] F.V. Tkachov, Phys. Lett. 100B (1981) 65;

K.G. Chetyrkin and F.V. Tkachov, Nucl. Phys. B192 (1981) 159.

[6] O.V. Tarasov, Phys. Rev. D54 (1996) 6479, Nucl. Phys. B502 (1997) 455.

[7] C. Anastasiou, E.W.N. Glover and C. Oleari, "Scalar one-loop integrals using the negative-dimension approach", hep-ph/9907494. To appear in Nucl. Phys. B.

[8] W.L. Van Neerven, Nucl. Phys. B268 (1986) 453.

[9] K.S. Kölbig, J.A. Mignaco and E. Remiddi, B.I.T. 10 (1970) 38. 